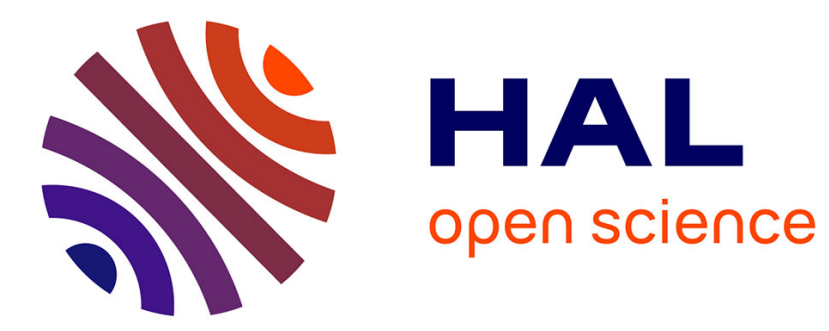

\title{
APPROXIMATE HEDGING IN A LOCAL VOLATILITY MODEL WITH PROPORTIONAL TRANSACTION COSTS
}

\author{
Emmanuel Lépinette, Tuan Tran Quoc
}

\section{- To cite this version:}

Emmanuel Lépinette, Tuan Tran Quoc. APPROXIMATE HEDGING IN A LOCAL VOLATILITY MODEL WITH PROPORTIONAL TRANSACTION COSTS. Applied Mathematical Finance, 2014, 21 (4), pp.313-341. hal-00687389

HAL Id: hal-00687389

https://hal.science/hal-00687389

Submitted on 15 Apr 2012

HAL is a multi-disciplinary open access archive for the deposit and dissemination of scientific research documents, whether they are published or not. The documents may come from teaching and research institutions in France or abroad, or from public or private research centers.
L'archive ouverte pluridisciplinaire HAL, est destinée au dépôt et à la diffusion de documents scientifiques de niveau recherche, publiés ou non, émanant des établissements d'enseignement et de recherche français ou étrangers, des laboratoires publics ou privés. 


\title{
APPROXIMATE HEDGING IN A LOCAL VOLATILITY MODEL WITH PROPORTIONAL TRANSACTION COSTS
}

\author{
By Emmanuel LEPINETTE*, Tuan TRAN* \\ CEREMADE, UMR CNRS 7534, \\ Paris-Dauphine University
}

\begin{abstract}
Local volatility models are popular because they can be simply calibrated to the market of European options. We extend the results of [4], [3] for such models, i.e. we propose a modified Leland method which allows us to approximately replicate a European contingent claim when the market is under proportional transaction costs.
\end{abstract}

1. Introduction. There are indications that the Black and Scholes model does not fit certain financial market phenomenons. In practice, the implied volatility is not constant, i.e. depends on the expiration date and the strike. This problem is known as the implied smile effect. More natural is then to consider local volatility models. Indeed, under mild assumptions, the strong Markov property and the continuity of the price process $S$ implies that $S$ is given by a local volatility function $\sigma\left(t, S_{t}\right)$. Bouchouev and Isakov [1] and later Egger, Hein and Hofmann [5] suggest to search for local volatility in the product form $\sigma(t, x)=\sigma(x) \rho(t)$. These local volatility models are very popular because they are simple to calibrate the call prices by the Dupire formula. In this paper, we consider a standard two-asset local volatility model in presence of transaction costs. Our goal is to provide a strategy which replicates approximately a European contingent claim $h\left(S_{T}\right)$ when the number of revision dates of the portfolio tends to $\infty$. We show that the Leland method can be adapted to the case of a local volatility model. This is a generalization of Papers [4] and [3] to the case where the volatility is not constant and depends on the underlying asset. The Leland strategy derives from the solution $\widehat{C}$ to the heat equation of the Black and Scholes model where we increase the volatility in order to compensate for the transaction costs. Contrarily to the case where the volatility is constant,

${ }^{*}$ The authors warmly express their thanks to Bruno Bouchard for his advices.

AMS 2000 subject classifications: 60G44, G11-G13

Keywords and phrases: Black-Scholes formula, transaction costs, Leland strategy, approximate hedging.

1

imsart-aap ver. 2011/05/20 file: Lepinette-Tran.tex date: April 13, 2012 
we do not have explicit expressions of the solution to the heat equation. The main difficulty is to obtain appropriate estimates of the derivatives of $\widehat{C}$, which is necessary to show the convergence of our scheme. To do so, we use PDEs techniques and our main concern is the case where the proportional transaction costs coefficient is constant, i.e. does not depend on the number $n$ of revision dates. For this model, the usual Leland method fails [4]. By modifying the Leland technique, we show that it is possible to obtain an approximate hedging of the pay-off $h\left(S_{1}\right)$ without limit error as $n \rightarrow \infty$ for a large class of pay-off functions $h$.

2. The model. We assume without loss of generality that the time horizon is $T=1$. The non-risky asset is the numéraire $S^{0}=1$ and the risky asset is given under the martingale probability measure by the SDE:

$$
d S_{t}=S_{t} \sigma\left(t, S_{t}\right) d W_{t}, \quad 0 \leq t \leq 1
$$

We suppose that trading involves proportional transaction costs with the transaction cost coefficients depending on the number of revision dates $n$ :

$$
k_{n}=k_{0} n^{-\alpha}, \quad \alpha \in\left[0, \frac{1}{2}\right] .
$$

The case $\alpha>0$ means that the transaction costs decreases as the number of times the agent rebalances his/her portfolio increases. This is the Leland model. If $\alpha=0$, the transaction costs coefficient is constant, i.e. does not depend on $n$. This model may be considered as more realistic and represents our main concern. Although, a transaction costs rate, e.g. $r=0.001$, can be also seen as $r=k_{0} n^{-\alpha}$ with $\alpha>0$ and $n$ large enough. In this case, $k_{0}$ depends on $\alpha$ and we leave the qualitative analysis of such approach for future research.

In our model the current value of the portfolio process at time $t$ is defined by

$$
V_{t}^{n}=V_{0}^{n}+\int_{0}^{t} D_{u}^{n} d S_{u}-\sum_{t_{i} \leq t} k_{n} S_{t_{i}}\left|D_{i+1}^{n}-D_{i}^{n}\right|, \quad t \leq 1 .
$$

where $t_{i}=t_{i}^{n}, 0 \leq i \leq n, t_{0}=0, t_{n}=1$, are the revision dates; and $D^{n}=D_{i}^{n}$ on the interval $\left.] t_{i-1}, t_{i}\right], D_{n+1}^{n}:=D_{n}^{n}$, where $D_{i}^{n}$ is $\mathcal{F}_{t_{i-1}}$-mesurable, i.e. $D^{n}$ is the trading strategy. The number of the risky asset that the holder possesses in the period $i$ is then $D_{i}^{n}$. The dynamics (2.1) means that the portfolio process $V^{n}$ is self-financed and in presence of transaction costs which are 
proportional to the traded volume. We suppose that the dates $t_{i}^{\prime} s$ are uniform i.e. $t_{i}=t_{i}^{n}=i / n, i=0, \ldots, n$.

In the complete model without friction, a contingent claim $h\left(S_{1}\right)$ is exactly replicated by the terminal value of the self-financed portfolio:

$$
V_{t}=E\left(h\left(S_{1}\right) \mid \mathcal{F}_{t}\right)=C\left(t, S_{t}\right)=V_{0}+\int_{0}^{t} C_{x}\left(r, S_{r}\right) d S_{r}, \quad t \leq 1
$$

where $C$ is solution of the PDE:

$$
\left(\mathbf{e}_{\mathbf{0}}\right)=\left\{\begin{array}{l}
\left.C_{t}(t, x)+\frac{1}{2} \sigma^{2}(t, x) x^{2} C_{x x}(t, x)=0, \quad(x, t) \in\right] 0, \infty[\times[0,1[ \\
C(1, x)=h(x), \quad x \in] 0, \infty[.
\end{array}\right.
$$

With transaction costs, we follow Leland's approach [9], i.e. we construct a strategy which can be considered as a modified Delta of Black-Scholes replication formula. The idea is to subtitute the volatility $\sigma$ by an enlarged one $\widehat{\sigma}(t, x)$ in order to compensate for the transaction costs. The "enlarged volatility" is defined by:

$$
\widehat{\sigma}^{2}(t, x)=\sigma^{2}(t, x)+\sigma(t, x) \gamma_{n},
$$

where

$$
\gamma_{n}=k_{n} n^{\frac{1}{2}} \sqrt{\frac{8}{\pi}}
$$

So, we introduce the PDE:

$$
(\mathbf{e})=\left\{\begin{array}{l}
\left.\widehat{C}_{t}(t, x)+\frac{1}{2} \widehat{\sigma}^{2}(t, x) x^{2} \widehat{C}_{x x}(t, x)=0, \quad(x, t) \in\right] 0, \infty[\times[0,1[, \\
\widehat{C}(1, x)=h(x), \quad x \in] 0, \infty[.
\end{array}\right.
$$

The existence of a solution to the PDE (e) is ensured by Lemma 5.2. Let us precise the intuition behind the Leland strategy. By the Ito Formula, assuming that the solution $\widehat{C}$ to (e) is smooth enough, we have

$$
\begin{aligned}
\widehat{C}\left(t, S_{t}\right)= & \widehat{C}\left(0, S_{0}\right)+\int_{0}^{t} \widehat{C}_{x}\left(u, S_{u}\right) d S_{u} \\
& +\frac{1}{2} \int_{0}^{t}\left[\sigma^{2}\left(u, S_{u}\right)-\widehat{\sigma}^{2}\left(u, S_{u}\right)\right] S_{u}^{2} \widehat{C}_{x x}\left(u, S_{u}\right) d u .
\end{aligned}
$$

Then, $\widehat{C}\left(t, S_{t}\right)$ can be seen as the continuous version of a portfolio process (2.1) provided that $D_{i}^{n}=\widehat{C}\left(t_{i-1}, S_{t_{i-1}}\right)$ and the drift term in the formula above corresponds to the cumulated transaction costs, i.e. we want to make equal the two following increments :

$$
\frac{1}{2}\left[\sigma^{2}\left(u, S_{u}\right)-\widehat{\sigma}^{2}\left(u, S_{u}\right)\right] S_{u}^{2} \widehat{C}_{x x}\left(u, S_{u}\right) \Delta u
$$

imsart-aap ver. 2011/05/20 file: Lepinette-Tran.tex date: April 13, 2012 
and

$$
-k_{0} n^{-\alpha}\left|\widehat{C}_{x}\left(u+\Delta u, S_{u+\Delta u}\right)-\widehat{C}_{x}\left(u, S_{u}\right)\right| S_{u+\Delta u} .
$$

To do so, we use the Taylor approximation

$$
\begin{aligned}
\widehat{C}_{x}\left(u+\Delta u, S_{u+\Delta u}\right)-\widehat{C}_{x}\left(u, S_{u}\right) & \simeq \widehat{C}_{x t}\left(u, S_{u}\right) \Delta_{u}+\widehat{C}_{x x}\left(u, S_{u}\right)\left(S_{u+\Delta u}-S_{u}\right), \\
& \simeq \widehat{C}_{x x}\left(u, S_{u}\right)\left(S_{u+\Delta u}-S_{u}\right)
\end{aligned}
$$

where

$$
S_{u+\Delta u}-S_{u} \simeq \sigma\left(u, S_{u}\right) S_{u}\left(W_{u+\Delta u}-W_{u}\right) .
$$

Assuming that $\widehat{C}_{x x} \geq 0$, we should thus look for $\widehat{\sigma}$ such that

$$
\frac{1}{2}\left[\sigma^{2}\left(u, S_{u}\right)-\widehat{\sigma}^{2}\left(u, S_{u}\right)\right] \Delta u \simeq-k_{0} n^{-\alpha} \sigma\left(u, S_{u}\right)\left|W_{u+\Delta u}-W_{u}\right| \frac{S_{u+\Delta u}}{S_{u}} .
$$

Then, considering the conditional expectation knowing $\mathcal{F}_{u}$, and the equalities

$$
E\left|W_{\Delta u}\right|=\sqrt{\Delta u} \sqrt{\frac{2}{\pi}}, \quad \frac{S_{u+\Delta u}}{S_{u}}=1+\sigma\left(u, S_{u}\right)\left(W_{u+\Delta u}-W_{u}\right) .
$$

we obtain, considering only the main terms, that

$$
\frac{1}{2}\left[\sigma^{2}\left(u, S_{u}\right)-\widehat{\sigma}^{2}\left(u, S_{u}\right)\right] \Delta u=-k_{0} n^{-\alpha} \sigma\left(u, S_{u}\right) \sqrt{\Delta_{u}} \sqrt{\frac{2}{\pi}} .
$$

We deduce that

$$
\widehat{\sigma}^{2}\left(u, S_{u}\right)=\sigma^{2}\left(u, S_{u}\right)+k_{0} n^{1 / 2-\alpha} \sqrt{\frac{8}{\pi}} \sigma\left(u, S_{u}\right) .
$$

We recall that the Leland strategy fails in the case $\alpha=0$, i.e. an approximation error appears. We propose a modified strategy as in [3] to treat the general case $\alpha \in\left[0, \frac{1}{2}\right]$ :

$$
\widehat{V}_{t}^{n}=\widehat{V}_{0}^{n}+\int_{0}^{t} \widehat{D}_{u}^{n} d S_{u}-\sum_{t_{i} \leq t} k_{n} S_{t_{i}}\left|\widehat{D}_{i+1}^{n}-\widehat{D}_{i}^{n}\right|, \quad 0 \leq t \leq 1,
$$

where $\widehat{V}_{0}^{n}=\widehat{C}_{x}^{n}\left(0, S_{0}\right)$, and $\widehat{D}_{t}^{n}=\widehat{D}_{t_{i}}^{n}$ on $\left.] t_{i-1}, t_{i}\right], t_{i} \leq 1, \widehat{D}_{t_{i}}^{n}$ is given by

$$
\begin{aligned}
& \quad(2.2) \widehat{D}_{t_{i}}^{n}=\widehat{C}_{x}^{n}\left(t_{i-1}, S_{t_{i-1}}\right)-\sum_{1 \leq j \leq i-1}\left[\widehat{C}_{x}^{n}\left(t_{j}, S_{t_{j-1}},\right)-\widehat{C}_{x}^{n}\left(t_{j-1}, S_{t_{j-1},}\right)\right] . \\
& \text { imsart-aap ver. } 2011 / 05 / 20 \text { file: Lepinette-Tran.tex date: April 13, } 2012
\end{aligned}
$$


We make use the abbreviations $\widehat{H}_{t}=\widehat{C}_{x}\left(t, S_{t}\right)=\widehat{C}_{x}^{n}\left(t, S_{t}\right)$ where we often omit the index $n, \widehat{h}_{t}=\widehat{C}_{x x}\left(t, S_{t}\right), \widehat{H}_{t_{i}}^{n}=\widehat{C}_{x}\left(t_{i}, S_{t_{i}}\right), t_{i}=t_{i}^{n}$, and

$$
K_{t}^{n}=\sum_{t_{i}^{n}<t} \Delta K_{t_{i}^{n}}^{n}
$$

where $\Delta K_{t_{0}^{n}}^{n}=0$ and for $i \leq 1$ :

$$
\Delta K_{t_{i}^{n}}^{n}=-\left[\widehat{C}_{x}\left(t_{j}, S_{t_{j-1}},\right)-\widehat{C}_{x}\left(t_{j-1}, S_{t_{j-1}},\right)\right]
$$

In this paper, we impose hypothesis on the pay-off function:

Assumption $(\mathbf{H}): h$ is a continuous function on $[0, \infty[$ which is differentiable except at the points $K_{1}<\cdots<K_{p}<\ldots$ where the first derivative $h^{\prime}$ admits right and left limits. Moreover, $h^{\prime}$ is bounded and $h$ has polynomial growth, i.e, $|h(x)| \leq M\left(1+x^{a}\right) \mid$ for some positive constants $M, a$.

In the case $\alpha=0$, we only consider pay-off functions $h$ behaving as affine functions at infinity. The pay-off functions in the European option contracts are generally of this form.

Assumption ( $\left.\mathbf{H}^{\prime}\right) h(x)=h_{1}(x)+a x+b$ where $h_{1}$ is a bounded continuous function.

Assumption (E): There exists some positive constant $K$ such that:

(a) $m \leq \sigma \leq M$ for some positive constants $M, m$.

(b) $\left|\sigma(t, x)-\sigma\left(t^{\prime}, x^{\prime}\right)\right| \leq K\left(\left|t-t^{\prime}\right|+\left|x-x^{\prime}\right|\right)$.

(c) $\frac{\partial^{k}}{\partial x^{k}} \sigma(t, x)$ and $\frac{\partial^{2}}{\partial x \partial t} \sigma(t, x)$ are continuous, and

$$
\left|x^{k} \frac{\partial^{k}}{\partial x^{k}} \sigma(t, x)\right|+\left|\frac{\partial^{2}}{\partial x \partial t} \sigma(t, x)\right| \leq K, \quad \forall 0 \leq k \leq 3 .
$$

Remark 2.1. Observe that Assumption (E) is satisfied by the local volatility functions $\sigma(t, x)=\gamma(t) e^{-c x^{2}}, \sigma(t, x)=\gamma(t)(\operatorname{atan}(x)+\pi)$, where $c>0$ and $\gamma$ is a bounded, positive and Lipschitz function. In the second case, the local volatility of the model increases as the price of the risky asset increases. The following example is given in [5]:

$$
\sigma(t, x)=\sqrt{\frac{1}{10}\left(1-\frac{1}{2} \exp \left(-4 \ln ^{2}(x)\right) \sin (2 \pi x)\right)} \sqrt{1+\frac{3}{5} \sin (2 \pi t)} .
$$

imsart-aap ver. 2011/05/20 file: Lepinette-Tran.tex date: April 13, 2012 
It is clear that Condition (a) holds. Since we have the inequalities

$$
\exp \left(-2 \ln ^{2}(x)\right) \leq \exp (2 \ln (x)) \leq x^{2}
$$

if $x \leq e^{-1}$, we easily deduce that the first derivative $\sigma_{x}(t, x)$ is uniformly bounded in $x$, hence Condition (b) holds.

Using the bounds $\exp \left(-4 \ln ^{2}(x)\right) \leq \exp (-4 \ln (x)) \leq x^{-4}$ if $x \geq e^{1}$, and the fact that $\ln ^{k}(x) \exp \left(-2 \ln ^{2}(x)\right)$ is bounded for all $k>0$, we also deduce that Condition (c) holds.

REMARK 2.2. Note that Condition (c) implies that $(t, x) \mapsto \sigma\left(t, e^{x}\right)$ is a Lipschitz function uniformly with respect to $(t, x) \in \mathbf{R}$ (since the first derivative of this function is bounded).

Our main result in this paper is the following convergence theorem.

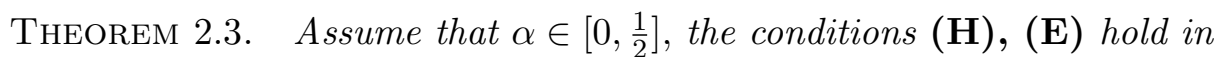
the case $\left.\alpha \in] 0, \frac{1}{2}\right]$, and the conditions $(\mathbf{H}),\left(\mathbf{H}^{\prime}\right)(\mathbf{E})$ hold in the case $\alpha=0$. Moreover, suppose that $h$ and $\sigma$ are such that $\widehat{C}_{x x} \geq 0$. If the strategy $\widehat{D}_{i}^{n}$ is given by (2.2), then the terminal value of the portfolio

$$
V_{1}^{n}=\widehat{C}_{0}^{n}+\int_{0}^{1} \widehat{D}_{u}^{n} d S_{u}-k_{n} \sum_{i=1}^{n-1} S_{t_{i}}\left|\widehat{D}_{i+1}^{n}-\widehat{D}_{i}^{n}\right|
$$

converges to the contingent claim $h\left(S_{1}\right)$ in probability.

REMARK 2.4. We can in fact show that the convergence still holds in the case $\alpha=0$ with only assumptions $\left(\mathbf{H}^{\prime}\right)$ and $(\mathbf{E})$. We add the condition (H) to this case just to avoid repeating all calculations for $\left.\alpha \in] 0, \frac{1}{2}\right]$.

REMARK 2.5. We do not need the portfolio rebalancing at the horizon date; that is why the cumulated sum of all transaction costs is taken only up to $n-1$. Notice that we need the condition $\widehat{C}_{x x} \geq 0$ to avoid the approximative error. In fact, this condition will not restrict so many our interesting cases. We refer to Lemma 5.6 for a sufficient condition ensuring the convexity of $C(t, x)$ in the variable $x$ and more generally to the papers [10] and [11] on the convexity propagation.

imsart-aap ver. 2011/05/20 file: Lepinette-Tran.tex date: April 13, 2012 
The paper is structured as follows. In Section 3, we state the proof of our main result, i.e. the convergence of the suggested terminal portfolio value to the pay-off. To do so, we need the upper bounds of the function $\widehat{C}(t, x)$ and its derivatives given in Section 4. Appendix is devoted to some auxiliary results used in the proofs.

3. Proof of the Theorem 2.3. By the Ito formula, we represent the difference $V_{1}^{n}-h\left(S_{1}\right)$ in a convenient form.

Lemma 3.1. We have $V_{1}^{n}-h\left(S_{1}\right)=F_{1}^{n}+F_{2}^{n}+F_{3}^{n}$ where

$$
\begin{aligned}
& F_{1}^{n}:=\int_{0}^{1}\left(H_{t}^{n}-\widehat{H}_{t}\right) d S_{t} \\
& F_{2}^{n}:=\frac{1}{2} \int_{0}^{1} \sigma\left(t, S_{t}\right) \gamma_{n} S_{t}^{2}\left|\widehat{C}_{x x}\left(t, S_{t}\right)\right| d t-k_{n} \sum_{i=1}^{n-1}\left|\Delta H_{t_{i}}^{n}+\Delta K_{t_{i}}^{n}\right| S_{t_{i}} \\
& F_{3}^{n}:=k_{n} \int_{0}^{1} K_{t}^{n} d S_{t}
\end{aligned}
$$

Our objective is to prove that $F_{1}^{n}, F_{2}^{n}, F_{3}^{n}$ converge to zero in probability. We will only separate two cases $\alpha=0$ and $\alpha \in] 0,1 / 2]$ in proving $F_{3}^{n} \rightarrow 0$ since $F_{1}^{n}, F_{2}^{n} \rightarrow 0$ without Condition (H'). Throughout the section, $C$ will designate a strictly positive constant independent of $n$ that could be changed from a line to another one. We put $\gamma_{n}=n^{1 / 2-\alpha}$.

Lemma 3.2. We have $P-\lim F_{1}^{n}=0$.

Proof Let us first consider the case $\alpha<\frac{1}{2}$. As $\widehat{C}_{x}(t, x)$ and $E\left(\sup _{u \in[0,1]} S_{u}^{2}\right)$ are bounded, the integral

$$
\int_{1-\frac{1}{\gamma_{n}}}^{1}\left(H_{t}^{n}-\widehat{H}_{t}\right) d S_{t}
$$

tends to 0 in $L^{2}$, so in probability. For each $t \in\left[t_{i-1}, t_{i}\left[, t<1-\frac{1}{\gamma_{n}}\right.\right.$, let us consider the Taylor expansion

$$
\widehat{C}_{x}\left(t_{i-1}, S_{t_{i-1}}\right)-\widehat{C}_{x}\left(t, S_{t}\right)=\widehat{C}_{x t}\left(\theta_{i}, S_{t_{i-1}}\right)\left(t_{i-1}-t\right)+\widehat{C}_{x x}\left(t, \widetilde{S}_{t}\right)\left(S_{t_{i-1}}-S_{t}\right)
$$

where $\theta_{i} \in\left[t_{i-1}, t\right]$ and $\widetilde{S}_{t} \in\left[S_{t_{i-1}}, S_{t}\right]$. Using Theorem 4.4, we obtain that:

$$
\left|\widehat{C}_{x}\left(t_{i-1}, S_{t_{i-1}}\right)-\widehat{C}_{x}\left(t, S_{t}\right)\right| \leq C\left|\frac{t-t_{i-1}}{1-t}\right|+C \frac{\left|S_{t_{i-1}}-S_{t}\right|}{\tilde{S}_{t} \sqrt{\gamma_{n}(1-t)}}
$$


As $\left|\Delta t_{i}\right| \leq \frac{C}{n}$ and $1-t \geq \frac{1}{\gamma_{n}}$, the first term tends to 0 . Moreover, since $\left(S_{t}\right)_{0 \leq t \leq 1}$ is strictly positive and continuous we deduce that the second term tends to 0 a.s. Hence

$$
\widehat{C}_{x}\left(t_{i-1}, S_{t_{i-1}}\right)-\widehat{C}_{x}\left(t, S_{t}\right) \rightarrow 0, \quad \text { a.s. }
$$

Keeping in mind that $E\left(\sup _{u \in[0,1]} S_{u}^{2}\right)$ and $\widehat{C}_{x}(t, x)$ are bounded, we get the convergence

$E\left(\int_{0}^{1-\frac{1}{\gamma_{n}}}\left(H_{t}^{n}-\widehat{H}_{t}\right) d S_{t}\right)^{2}=\int_{0}^{1-\frac{1}{\gamma_{n}}} E\left[S_{t}^{2}\left(H_{t}^{n}-\widehat{H}_{t}\right)^{2}\right] d t \rightarrow 0 \quad$ as $\quad n \rightarrow \infty$

by virtue of the Lebesgue dominated convergence theorem. Hence we have shown that $\lim E\left(F_{1}^{n}\right)^{2}=0$ with $\alpha<\frac{1}{2}$. The case $\alpha=\frac{1}{2}$ is obivious because $\widehat{C}$ does not depend on $n$.

Let us now show that $F_{2}^{n} \longrightarrow 0$. By the Ito Formula, we have

$$
\widehat{C}_{x}\left(t, S_{t}\right)=\widehat{C}_{x}\left(0, S_{0}\right)+M_{t}^{n}+A_{t}^{n}
$$

where

$$
\begin{aligned}
M_{t}^{n} & :=\int_{0}^{t} \sigma\left(u, S_{u}\right) S_{u} \widehat{C}_{x x}\left(u, S_{u}\right) d W_{u}, \\
A_{t}^{n} & :=\int_{0}^{t}\left[\widehat{C}_{x t}\left(u, S_{u}\right)+\frac{1}{2} \sigma^{2}\left(u, S_{u}\right) S_{u}^{2} \widehat{C}_{x x x}\left(u, S_{u}\right)\right] d u .
\end{aligned}
$$

We write $F_{2}^{n}=\sum_{i=1}^{5} L_{i}$ where

$$
\begin{aligned}
& L_{1}^{n}:=\frac{1}{2} \int_{0}^{1} \sigma\left(t, S_{t}\right) \gamma_{n} S_{t}^{2} \widehat{h}_{t} d t-\frac{1}{2} \int_{0}^{1} \sum_{i=1}^{n-1} \sigma\left(t_{i-1}, S_{t_{i-1}}\right) \gamma_{n} S_{t_{i-1}}^{2} \widehat{h}_{t_{i-1}} I_{\left.t_{i-1}, t_{i}\right]}(t) d t \\
& L_{2}^{n}: \sum_{i=1}^{n-1} \sigma\left(t_{i-1}, S_{t_{i-1}}\right) \widehat{h}_{t_{i-1}} S_{t_{i-1}}^{2}\left(\frac{1}{2} \gamma_{n} \Delta t_{i}-k_{n} n^{1 / 2} \sqrt{\Delta t_{i}}\left|\Delta W_{t_{i}}\right|\right), \\
& L_{3}^{n}:=k_{n} \sum_{i=1}^{n-1} \sigma\left(t_{i-1}, S_{t_{i-1}}\right) S_{t_{i-1}}^{2} \widehat{h}_{t_{i-1}} n^{1 / 2} \sqrt{\Delta t_{i}}\left|\Delta W_{t_{i}}\right|-k_{n} \sum_{i=1}^{n-1} S_{t_{i-1}}\left|\Delta M_{t_{i}}\right|, \\
& L_{4}^{n}:=k_{n} \sum_{i=1}^{n-1} S_{t_{i-1}}\left|\Delta M_{t_{i}}\right|-k_{n} \sum_{i=1}^{n-1} S_{t_{i-1}}\left|\Delta H_{t_{i}}+\Delta K_{t_{i}}\right|, \\
& L_{5}^{n}:=-k_{n} \sum_{i=1}^{n-1} \Delta S_{t_{i}}\left|\Delta H_{t_{i}}+\Delta K_{t_{i}}\right|
\end{aligned}
$$


Lemma 3.3. We have $P-\lim L_{1}^{n}=0$.

Proof. We rewrite

$$
L_{1}^{n}=\frac{1}{2} \int_{t_{n-1}}^{1} \sigma\left(t, S_{t}\right) \gamma_{n} S_{t}^{2} \widehat{h}_{t} d t+\sum_{k=1}^{6} L_{1 k}^{n} .
$$

It is obvious that the first integral tends to 0 as $n$ tends to $\infty$. We now show that $L_{1 k}^{n} \rightarrow 0, \forall k$. These terms are defines as follows.

$$
L_{11}^{n}:=\frac{1}{2} \int_{0}^{1} \sum_{i=1}^{n-1}\left[\sigma\left(t, S_{t}\right)-\sigma\left(t_{i-1}, S_{t}\right)\right] \gamma_{n} S_{t}^{2} \widehat{h}_{t} I_{] t_{i-1}, t_{i}\right]}(t) d t .
$$

Using the hypothesis on $\sigma$, and Theorem 4.4 , we deduce a constant $c_{\omega}$ depending on $\omega \in \Omega$ such that:

$$
\begin{gathered}
L_{11}^{n} \leq \frac{c_{\omega}}{n} \int_{0}^{t_{n-1}} \frac{\sqrt{\gamma_{n}}}{\sqrt{1-t}} \leq c_{\omega} n^{-\frac{3}{4}-\frac{\alpha}{2}} \rightarrow 0 . \\
L_{12}^{n}:=\frac{1}{2} \int_{0}^{1} \sum_{i=1}^{n-1}\left[\sigma\left(t_{i-1}, S_{t}\right)-\sigma\left(t_{i-1}, S_{t_{i-1}}\right)\right] \gamma_{n} S_{t}^{2} \widehat{h}_{t} I_{\left[t_{i-1}, t_{i}\right]}(t) d t .
\end{gathered}
$$

Similarly, there is a constant $c_{\omega}$ depending on $\omega \in \Omega$ such that $\left|L_{12}^{n}\right| \leq c_{\omega} \widetilde{L}_{12}^{n}$, where

$$
\widetilde{L}_{12}^{n}=\sqrt{\gamma_{n}} \int_{0}^{t_{n-1}} \sum_{i=1}^{n-1} \frac{\left|S_{t}-S_{i-1}\right|}{\sqrt{1-t}} I_{] t_{i-1}, t_{i}\right]}(t) d t .
$$

Since $E\left|S_{t}-S_{i-1}\right| \leq C \sqrt{t-t_{i}}$ we obtain that

$$
E\left(\widetilde{L}_{12}^{n}\right) \leq C n^{-\frac{1}{4}-\frac{\alpha}{2}} \rightarrow 0 .
$$

Hence $L_{12}^{n} \rightarrow 0$ in probability.

$$
L_{13}^{n}:=\frac{1}{2} \int_{0}^{1} \sum_{i=1}^{n-1}\left[S_{t}^{2}-S_{t_{i-1}}^{2}\right] \gamma_{n} \sigma\left(t_{i-1}, S_{t_{i-1}}\right) \widehat{h}_{t} I_{] t_{i-1}, t_{i}\right]}(t) d t .
$$

A reasoning similar to the one used for $L_{12}^{n}$ leads to $P-\left|L_{13}^{n}\right| \rightarrow 0$.

$$
\begin{aligned}
& L_{14}^{n}:=\frac{1}{2} \int_{0}^{1} R_{t}^{n} d t \\
& R_{t}^{n}:=\sum_{i=1}^{n-1}\left[\left|\widehat{C}_{x x}\left(t, S_{t}\right)\right|-\left|\widehat{C}_{x x}\left(t, S_{t_{i-1}}\right)\right|\right] \gamma_{n} \sigma\left(t_{i-1}, S_{t_{i-1}}\right) S_{t_{i-1}}^{2} I_{\left.t_{i-1}, t_{i}\right]}(t) d t . \\
& \quad \text { imsart-aap ver. } 2011 / 05 / 20 \text { file: Lepinette-Tran.tex date: April 13, } 2012
\end{aligned}
$$


Using the inequality ||$a|-| b|| \leq|a-b|$ and the Taylor expansion

$$
\widehat{C}_{x x}\left(t, S_{t}\right)-\widehat{C}_{x x}\left(t, S_{t_{i-1}}\right)=\widehat{C}_{x x x}\left(t, \widetilde{S}_{i}\right)\left(S_{t}-S_{t_{i-1}}\right)
$$

where $\widetilde{S}_{i} \in\left[S_{t}, S_{t_{i-1}}\right]$, we deduce a.s. a constant $c_{\omega}$ such that $\left|L_{15}^{n}\right| \leq c_{\omega} \widetilde{L}_{15}^{n}$ with

$$
\widetilde{L}_{15}^{n}:=\int_{0}^{t_{n-1}} \sum_{i=1}^{n-1} \gamma_{n}\left|S_{t}-S_{t_{i-1}}\right|\left(\frac{1}{\sqrt{\gamma_{n}(1-t)}}+\frac{1}{\gamma_{n}(1-t)}\right) I_{] t_{i-1}, t_{i}\right]}(t) d t .
$$

Since $E\left|S_{t}-S_{t_{i-1}}\right| \leq C \sqrt{\Delta t_{i}}$, we get that

$$
\begin{gathered}
E \widetilde{L}_{15}^{n} \leq \frac{C}{\sqrt{n}} \int_{0}^{t_{n-1}}\left(\frac{\sqrt{\gamma_{n}}}{\sqrt{1-t}}+\frac{1}{1-t}\right) d t \\
\leq \quad C n^{-\frac{1}{4}-\frac{\alpha}{2}}+C \frac{\ln n}{\sqrt{n}} \rightarrow 0 . \\
L_{16}^{n}:=\frac{1}{2} \int_{0}^{1} \sum_{i=1}^{n-1}\left[\left|\widehat{C}_{x x}\left(t, S_{t_{i-1}}\right)\right|-\left|\widehat{C}_{x x}\left(t_{i-1}, S_{t_{i-1}}\right)\right|\right] \gamma_{n} \sigma\left(t_{i-1}, S_{t_{i-1}}\right) S_{t_{i-1}}^{2} I_{] t_{i-1}, t_{i}\right]}(t) d t .
\end{gathered}
$$

We use the same arguments as for $L_{15}^{n}$. The Taylor expansion yields:

$$
\widehat{C}_{x x}\left(t, S_{t_{i-1}}\right)-\widehat{C}_{x x}\left(t_{i-1}, S_{t_{i-1}}\right)=\widehat{C}_{x x t}\left({\widetilde{t_{i}}}_{i}, S_{t_{i-1}}\right)\left(t-t_{i-1}\right)
$$

where $\widetilde{t}_{i} \in\left[t_{i-1}, t_{i}\right]$. Using Theorem 4.4, we deduce that, for some constant $c_{\omega},\left|L_{16}^{n}\right| \leq c_{\omega} \widetilde{L}_{16}^{n}$ where

$$
\widetilde{L}_{16}^{n}=\int_{0}^{t_{n-1}} \frac{\gamma_{n}^{2}}{n}\left(\frac{1}{\sqrt{\gamma_{n}^{3}(1-t)^{3}}}\right) d t
$$

This integral tends to 0 as $n \rightarrow \infty$, i.e. $P-\lim L_{16}^{n}=0$.

Lemma 3.4. We have $P-\lim L_{2}^{n}=0$.

Proof. By hypothesis there exists a.s. a constant $c_{\omega}$ such that

$$
L_{2}^{n} \leq c_{\omega} \sum_{i=1}^{n-1}\left|\widehat{C}_{x x}\left(t_{i-1}, S_{t_{i-1}}\right)\right| S_{t_{i-1}} \xi_{i}
$$


APPROXIMATE HEDGING IN LOCAL VOLATILITY MODEL WITH FRICTION1

where

$$
\xi_{i}=\frac{1}{2} \gamma_{n} \Delta t_{i}-k_{n} n^{1 / 2} \sqrt{\Delta t_{i}}\left|\Delta W_{t_{i}}\right|
$$

is independent of $\mathcal{F}_{t_{i-1}}$, verifies $E \xi_{i}=0$, and $E \xi_{i}^{2}=k_{n}^{2} n\left(\Delta t_{i}\right)^{2}$. Using Theorem 4.4 we obtain that $L_{2}^{n} \leq c_{\omega} \widetilde{L}_{2}^{n}$ where

$$
\widetilde{L}_{2}^{n}:=\sum_{i=1}^{n-1} \frac{\xi_{i}}{\rho_{t_{i-1}}}
$$

By independence, we have

$$
\begin{aligned}
E\left(\widetilde{L}_{2}^{n}\right)^{2} & \leq \sum_{i=1}^{n-1} \frac{E \xi_{i}^{2}}{\rho_{t_{i-1}}^{2}} \\
& \leq \frac{n k_{n}^{2}\left(\Delta t_{i}\right)^{2}}{\gamma_{n}\left(1-t_{i-1}\right)} \\
& \leq C n^{-\alpha-\frac{1}{2}} \sum_{i=1}^{n-1} \frac{\Delta t_{i}}{1-t_{i-1}} .
\end{aligned}
$$

The sum $\sum_{i=1}^{n-1} \frac{\Delta t_{i}}{1-t_{i-1}}$ can be approximated by $\int_{0}^{t_{n-1}} \frac{d t}{1-t}$ which is $O(\ln n)$. It then follows that $\widetilde{L}_{2}^{n} \rightarrow 0$ in $L^{2}$ and then $L_{2}^{n} \rightarrow 0$ in probability.

Lemma 3.5. We have $P-\lim L_{3}^{n}=0$.

Proof. Let us write $\left|L_{3}^{n}\right| \leq D_{1}^{n}+D_{2}^{n}+D_{3}^{n}$ where $D_{i}^{n}, i=1,2,3$, are defined below.

$$
D_{1}^{n}=k_{n} \sum_{i=1}^{n-1}\left|\int_{t_{i-1}}^{t_{i}} \xi_{i}(t) d W_{t}\right|
$$

with

$$
\xi_{i}(t)=S_{t_{i-1}}^{2}\left[\sigma\left(t_{i-1}, S_{t_{i-1}}\right)-\sigma\left(t_{i-1}, S_{t}\right)\right] \widehat{C}_{x x}\left(t_{i-1}, S_{t_{i-1}}\right) .
$$

We immediately get that

$$
\left\|D_{1}^{n}\right\|_{2} \leq C n^{-\alpha} \sum_{i=1}^{n-1}\left(\int_{t_{i-1}}^{t_{i}} E \xi_{i}^{2}(t) d t\right)^{1 / 2} .
$$

Using the hypothesis on $\sigma$ and Theorem 4.4, we obtain that:

$$
\left\|D_{1}^{n}\right\|_{2} \leq c n^{-\alpha} \sum_{i=1}^{n-1}\left(\int_{t_{i-1}}^{t_{i}} \frac{\Delta t_{i} d t}{\gamma_{n}\left(1-t_{i-1}\right)}\right)^{1 / 2} .
$$

imsart-aap ver. 2011/05/20 file: Lepinette-Tran.tex date: April 13, 2012 
Hence

$$
\left\|D_{1}^{n}\right\|_{2} \leq c n^{-\frac{\alpha}{2}-\frac{1}{4}} \sum_{i=1}^{n-1} \frac{\Delta t_{i}}{\sqrt{1-t_{i-1}}} \rightarrow 0
$$

We have

$$
D_{2}^{n}=k_{n} \sum_{i=1}^{n-1}\left|\int_{t_{i-1}}^{t_{i}} \widetilde{\xi}_{i}(t) d W_{t}\right|
$$

where

$$
\widetilde{\xi}_{i}(t)=S_{t_{i-1}}^{2}\left[\sigma\left(t_{i-1}, S_{t}\right)-\sigma\left(t, S_{t}\right)\right] \widehat{C}_{x x}\left(t_{i-1}, S_{t_{i-1}}\right) .
$$

Using he hypothesis on $\sigma$ and Theorem 4.4, we also deduce that $\left\|D_{2}^{n}\right\|_{2} \rightarrow 0$.

The last term is

$$
D_{3}^{n}=k_{n} \sum_{i=1}^{n-1}\left|\int_{t_{i-1}}^{t_{i}} \mathcal{X}_{i}(t) d W_{t}\right|
$$

where

$$
\mathcal{X}_{i}(t)=\left[S_{t_{i-1}} \widehat{C}_{x x}\left(t_{i-1}, S_{t_{i-1}}\right)-S_{t} \widehat{C}_{x x}\left(t, S_{t}\right)\right] \sigma\left(t, S_{t}\right) .
$$

We first observe that

$$
\left\|D_{3}^{n}\right\|_{2} \leq C n^{-\alpha} \sum_{i=1}^{n-1}\left(\int_{t_{i-1}}^{t_{i}} E \mathcal{X}_{i}^{2}(t) d t\right)^{1 / 2}
$$

Using the Ito formula

$$
d\left[S_{t} \widehat{h}^{t}\right]=d\left[S_{t} \widehat{C}_{x x}\left(t, S_{t}\right)\right]=f_{t} d W_{t}+g_{t} d t
$$

where

$$
\begin{gathered}
f_{t}:=\sigma S_{t} \widehat{C}_{x x}\left(t, S_{t}\right)+\sigma S_{t}^{2} \widehat{C}_{x x x}\left(t, S_{t}\right) \\
g_{t}:=S_{t} \widehat{C}_{x x t}\left(t, S_{t}\right)+\frac{1}{2} \sigma^{2} S_{t}^{3} \widehat{C}_{x x x x}\left(t, S_{t}\right)+\sigma^{2} S_{t}^{2} \widehat{C}_{x x x}\left(t, S_{t}\right),
\end{gathered}
$$

we deduce that

$$
E\left(S_{t} \widehat{h}^{t}-S_{t_{i-1}} \widehat{h}_{t_{i-1}}\right)^{2} \leq 2 \int_{t_{i-1}}^{t_{i}} E\left(f_{u}^{2}\right) d u+2 \Delta t_{i} \int_{t_{i-1}}^{t_{i}} E\left(g_{u}^{2}\right) d u
$$

It follows that

$$
\begin{gathered}
\left(\int_{t_{i-1}}^{t_{i}} E \mathcal{X}_{i}^{2}(t) d t\right)^{1 / 2} \leq \sqrt{2 \Delta t_{i}}\left(\int_{t_{i-1}}^{t_{i}} E\left(f_{u}^{2}\right) d u\right)^{\frac{1}{2}}+\Delta t_{i}\left(2 \int_{t_{i-1}}^{t_{i}} E\left(g_{u}^{2}\right) d u\right)^{\frac{1}{2}} \\
\quad \text { imsart-aap ver. } 2011 / 05 / 20 \text { file: Lepinette-Tran.tex date: April 13, } 2012
\end{gathered}
$$


APPROXIMATE HEDGING IN LOCAL VOLATILITY MODEL WITH FRICTION3

By Theorem 4.4, we get the bounds

$$
\begin{aligned}
E\left(\left|D_{3}^{n}\right|\right) & \leq C n^{-\alpha} \sum_{i=1}^{n-1}\left(\frac{\Delta t_{i}}{\rho_{t_{i-1}}}+\frac{\Delta t_{i}}{\rho_{t_{i-1}}^{2}}\right)+C n^{-1 / 2-\alpha} \sum_{i=1}^{n-1} \frac{\left(\Delta t_{i}\right) \gamma_{n}}{\rho_{t_{i-1}}^{3}} \\
& \leq C n^{-\frac{1}{2}} \ln n+C n^{-1 / 4-\alpha / 2} .
\end{aligned}
$$

where $C$ is a constant. It follows that $E\left(\left|D_{3}^{n}\right|\right)$ converges to 0 .

Lemma 3.6. We have $P-\lim L_{4}^{n}=0$.

Proof. We first show that we may replace $\Delta K_{t_{i}}$ by

$$
\Delta \tilde{K}_{t_{i}}:=-\int_{t_{i-1}}^{t_{i}} C_{x t}\left(u, S_{u}\right) d u .
$$

To do so, it suffices to show that $\chi^{n} \rightarrow 0$ where

$$
\chi^{n}:=k_{n} \sum_{i \leq n-1} S_{t_{i-1}} \int_{t_{i-1}}^{t_{i}}\left(\widehat{C}_{x t}\left(u, S_{u}\right)-\widehat{C}_{x t}\left(u, S_{t_{i-1}}\right)\right) d u .
$$

Using a Taylor expansion

$$
\widehat{C}_{x t}\left(u, S_{u}\right)-\widehat{C}_{x t}\left(u, S_{t_{i-1}}\right)=\widehat{C}_{x x t}\left(u, \bar{S}_{t_{i-1}}\right)\left(S_{u}-S_{t_{i-1}}\right)
$$

we deduce that $\chi^{n} \leq c_{\omega} \bar{\chi}^{n}$ where, by Theorem 4.4 ,

$$
\bar{\chi}^{n}:=k_{n} \gamma_{n} \sum_{i=1}^{n-1} \int_{t_{i-1}}^{t_{i}} \frac{S_{u}-S_{t_{i-1}}}{(1-t)^{3 / 2} \gamma_{n}^{3 / 2}} d u .
$$

As $E\left|S_{u}-S_{t_{i-1}}\right| \leq c \sqrt{\Delta t_{i}}$, we easily conclude that $E \bar{\chi}^{n} \rightarrow 0$.

Using the inequality ||$a|-| b|| \leq|a-b|$ and assuming that $\Delta K_{t_{i}}=\Delta \tilde{K}_{t_{i}}$, we get that

$$
\left|L_{n}^{4}\right| \leq k_{n} \sum_{i=1}^{n-1} S_{t_{i-1}}\left|\Delta A_{t_{i}}+\Delta K_{t_{i}}\right| \leq c(\omega) k_{n} \int_{0}^{t_{n-1}} \sigma^{2} S_{u}^{2}\left|\widehat{C}_{x x x}\left(u, S_{u}\right)\right| d u .
$$

By virtue of Theorem 4.4, we get that:

$$
\left|L_{n}^{4}\right| \leq c(\omega) k_{n} \int_{0}^{t_{n-1}}\left(\frac{1}{\sqrt{\gamma_{n}(1-t)}}+\frac{1}{\gamma_{n}(1-t)}\right) d t \rightarrow 0
$$

imsart-aap ver. 2011/05/20 file: Lepinette-Tran.tex date: April 13, 2012 
Lemma 3.7. We have $P-\lim L_{5}^{n}=0$.

Proof. We use similar arguments. First, using a Taylor expansion and Theorem 4.6, we deduce that

$$
A^{n}:=k_{n} \sum_{i=1}^{n-1} \Delta S_{t_{i}}\left|\Delta H_{t_{i}}\right| \leq c_{\omega} k_{n} \sum_{i=1}^{n-1}\left[\frac{\left(\Delta S_{t_{i}}\right)^{2}}{\sqrt{\gamma_{n}\left(1-t_{i}\right)}}+\frac{\Delta t_{i} \Delta S_{t_{i}}}{\left(1-t_{i}\right)}\right] .
$$

As $E\left(\Delta S_{t_{i}}\right)^{2} \leq C \Delta t_{i}$, we deduce that $A^{n} \rightarrow 0$. Secondly, as in Lemma 3.6 , we may assume without loss of generality that $\Delta K_{t_{i}}=\Delta \tilde{K}_{t_{i}}$. Therefore, it remains to estimate, by virtue of Theorem 4.4

$$
B^{n}:=k_{n} \sum_{i=1}^{n-1} \Delta S_{t_{i}}\left|\Delta \tilde{K}_{t_{i}}\right| \leq c_{\omega} k_{n} \sum_{i=1}^{n-1} \int_{t_{i-1}}^{t_{i}} \frac{\Delta S_{t_{i}}}{1-u} d u
$$

We easily get that $B^{n} \rightarrow 0$.

Lemma 3.8. We have $P-\lim F_{3}^{n}=0$.

Proof. Let us define $\bar{F}_{3}^{n}:=\int_{0}^{1} K_{t}^{n} d S_{t}$. Observe that $\bar{F}_{3}^{n}$ can be rewritten as

$\bar{F}_{3}^{n}=\sum_{i=1}^{n} K_{t_{i}}^{n} \Delta S_{t_{i}}=-\sum_{i=1}^{n-1} S_{t_{i}} \Delta K_{t_{i}}^{n}+K_{1}^{n} S_{1}-K_{t_{1}}^{n} S_{0}=-\sum_{i=1}^{n-1} S_{t_{i}} \Delta K_{t_{i}}^{n}+K_{1}^{n} S_{1}$

Since $F_{3}^{n}=k_{n} \bar{F}_{3}^{n}$, we assume without loss of generality that $\Delta K_{t_{i}}=\Delta \tilde{K}_{t_{i}}$ as shown in Lemma 3.6. Therefore,

$$
\begin{aligned}
\bar{F}_{3}^{n} & =\sum_{i=1}^{n-1} \int_{t_{i-1}}^{t_{i}} \widehat{C}_{t x}\left(u, S_{u}\right) S_{t_{i}} d u-S_{1} \int_{0}^{t_{n-1}} \widehat{C}_{t x}\left(u, S_{u}\right) d u \\
& =\int_{0}^{t_{n-1}} \widehat{C}_{t x}\left(u, S_{u}\right)\left(S_{u}-S_{1}\right) d u+\sum_{i=1}^{n-1} \int_{t_{i-1}}^{t_{i}} \widehat{C}_{t x}\left(u, S_{u}\right)\left(S_{t_{i}}-S_{u}\right) d u
\end{aligned}
$$

Note that $E\left|S_{u}-S_{t}\right| \leq C \sqrt{|t-u|} \leq C \sqrt{|1-u|}, \quad \forall u, t$, and recall that $E\left(\sup _{0 \leq u \leq 1} S_{u}\right)<\infty$. Let us now separate the analysis in two cases: $0 \leq u \leq 1$

- $\alpha \in] 0,1 / 2]$. We use the bound $\left|\widehat{C}_{x t}(t, x)\right| \leq \frac{C}{1-t}$ and we deduce that

$$
E\left|F_{3}^{n}\right| \leq c n^{-\alpha} \int_{0}^{t_{n-1}} \sqrt{1-u} \frac{1}{1-u} d u \leq c n^{-\alpha} \rightarrow 0 .
$$


- $\alpha=0$. In this case we use Lemma 4.5. We get that

$$
E\left|F_{3}^{n}\right| \leq c \int_{0}^{t_{n-1}} \frac{1}{\sqrt{\gamma_{n}}(1-t)} d u \leq c n^{-1 / 4} \ln n \rightarrow 0 .
$$

Therefore, we have shown that $P-\lim F_{3}^{n}=0$.

4. Estimation of the Derivatives of $\widehat{C}(t, x)$. Before analysing the function $\widehat{C}(t, x)$ and getting some upper bounds for its derivatives, we are going to use the time change technique to reduce our problem into the case where the diffusion coefficient in (e) is bounded independently of $n$. We then give an explicit expression of $\widehat{C}(t, x)$ and $\widehat{C}_{x}(t, x)$ and we deduce estimates of the derivatives. To do so, we utilize the analysis of the fundamental solution associated to a parabolic-type PDE given in [7] with the change of variable $x=e^{y}, y \in \mathbf{R}$. Notice that Friedman imposes that the coefficients of the parabolic-type PDE are Holder continuous of exponent $0<\beta<1$. In fact, the uniform Lipschitz condition is sufficient as shown in [2] but the conditions we impose on $\sigma$ implies that the mapping $x \mapsto \widehat{\sigma}^{2}\left(t, e^{x}\right)$ is Holder continuous whatever the exponent $0<\beta<1$. In all this section, we suppose that the assumptions $(\mathbf{E}),(\mathbf{H})$ hold. We shall pay special attention to the case $\alpha=0$ with Assumption $\left(\mathbf{H}^{\prime}\right)$. This is the most difficult case of our analysis for which we need specific bounds of the derivatives of $\widehat{C}$.

Recall that $\widehat{C}(t, x)$ satisfies the equation

$$
\left\{\begin{array}{l}
\left.\widehat{C}_{t}(t, x)+\frac{1}{2} \widehat{\sigma}^{2}(t, x) x^{2} \widehat{C}_{x x}(t, x)=0, \quad(x, t) \in\right] 0, \infty[\times[0,1[, \\
\widehat{C}(1, x)=h(x), \quad x \in] 0, \infty[.
\end{array}\right.
$$

Consider the change of time $s:=t \gamma_{n}$. Then, $u(s, x):=\widehat{C}\left(\frac{s}{\gamma_{n}}, x\right)$ satisfies the following PDE:

$(\mathbf{f})=\left\{\begin{array}{ccc}\widehat{u}_{t}(t, x) & + & \left.\frac{1}{2}\left(\widehat{\sigma}^{*}\right)^{2}(t, x) x^{2} \widehat{u}_{x x}(t, x)=0, \quad(x, t) \in\right] 0, \infty\left[\times\left[0, \gamma_{n}[,\right.\right. \\ \widehat{u}\left(\gamma_{n}, x\right)= & h(x), \quad x \in] 0, \infty[,\end{array}\right.$

where $\left(\widehat{\sigma}^{*}\right)^{2}(t, x)=\frac{\widehat{\sigma}^{2}(t, x)}{\gamma_{n}}$. We easily check that $\widehat{\sigma}^{*}(s, x)$ satisfies Assumption $(\mathbf{E})$ for some constants which do not dependent of $n$. Using the definition of $u$, it can be shown that

$$
\frac{\partial^{k}}{\partial x^{k}} \frac{\partial^{r}}{\partial s^{r}} u(s, x)=\frac{1}{\gamma_{n}^{r}} \frac{\partial^{k}}{\partial x^{k}} \frac{\partial^{r}}{\partial t^{r}} C(t, x) .
$$

imsart-aap ver. 2011/05/20 file: Lepinette-Tran.tex date: April 13, 2012 
According to the proof of Lemma 5.2, the unique solution of (f) is given by

$$
\widehat{u}(s, x)=\operatorname{Eh}\left(\widehat{S}_{s, x}\left(\gamma_{n}\right)\right)
$$

where $\widehat{S}_{s, x}$ is solution of the PDE

$$
(\hat{\mathbf{S}})=\left\{\begin{array}{c}
\left.\left.d \widehat{S}_{s, x}(u)=\widehat{\sigma}^{*}\left(u, \widehat{S}_{s, x}(u)\right) \widehat{S}_{s, x}(u) d W_{u}, \quad u \in\right] s, \gamma_{n}\right] \\
\widehat{S}_{s, x}(s)=x
\end{array}\right.
$$

We need the probabilistic representation (4.2) of $\widehat{u}(s, x)$ in the case $\alpha=0$. If $\alpha>0$, we use the following representation of $\widehat{u}_{x}(s, x)$. Let us define

$$
\widehat{\Lambda}^{*}(t, x):=\left(\widehat{\sigma}^{*}(t, x)+x \widehat{\sigma}_{x}^{*}(t, x)\right) \widehat{\sigma}^{*}(t, x)
$$

and, by Lemma 5.3 , we consider $\widetilde{S}_{x, t}$ the solution of the eds:

$\left\{\begin{aligned} d \widetilde{S}_{x, t}(u) & \left.\left.=\widehat{\sigma}^{*}\left(u, \widetilde{S}_{x, t}(u)\right) \widetilde{S}_{x, t}(u) d W_{u}+\widehat{\Lambda}^{*}\left(u, \widetilde{S}_{x, t}(u)\right) \widetilde{S}_{x, t}(u) d u, \quad u \in\right] s, \gamma_{n}\right] \\ \widetilde{S}_{x, t}(t) & =x\end{aligned}\right.$

Then, we have:

LEMMA 4.1.

$$
\widehat{u}_{x}(s, x)=E h^{\prime}\left(\widetilde{S}_{s, x}\left(\gamma_{n}\right)\right)
$$

Proof. We write:

$$
\begin{aligned}
\widehat{u}(t, x)-\widehat{u}\left(t, x_{0}\right) & =\operatorname{Eh}\left(\widehat{S}_{x, t}\left(\gamma_{n}\right)\right)-E h\left(\widehat{S}_{x_{0}, t}\left(\gamma_{n}\right)\right) \\
\widehat{u}(t, x)-\widehat{u}\left(t, x_{0}\right) & =E \int_{0}^{\gamma_{n}} \frac{d}{d \mu} h\left(\widehat{S}_{x_{0}, t}\left(\gamma_{n}\right)+\mu\left(\widehat{S}_{x, t}\left(\gamma_{n}\right)-\widehat{S}_{x_{0}, t}\left(\gamma_{n}\right)\right)\right) d \mu .
\end{aligned}
$$

Since $h^{\prime}$ exists out of a countable set, $\left(\widehat{C}(t, x)-\widehat{C}\left(t, x_{0}\right)\right) /\left(x-x_{0}\right)$ is equal to

$$
E \int_{0}^{\gamma_{n}} h^{\prime}\left(\widehat{S}_{x_{0}, t}\left(\gamma_{n}\right)+\mu\left(\widehat{S}_{x, t}\left(\gamma_{n}\right)-\widehat{S}_{x_{0}, t}\left(\gamma_{n}\right)\right) \frac{\widehat{S}_{x, t}\left(\gamma_{n}\right)-\widehat{S}_{x_{0}, t}\left(\gamma_{n}\right)}{x-x_{0}} d \mu .\right.
$$

As Assumption (E) holds, we apply Theorem 5.12 p120 [6] and deduce that $\frac{\partial \widehat{S}_{x, t}\left(\gamma_{n}\right)}{\partial x}$ exists in the $L^{2}$ sense, i.e.:

$$
\frac{\widehat{S}_{x, t}\left(\gamma_{n}\right)-\widehat{S}_{x_{0}, t}\left(\gamma_{n}\right)}{x-x_{0}} \rightarrow \frac{\partial \widehat{S}_{x_{0}, t}\left(\gamma_{n}\right)}{\partial x} \text { in } L^{2}
$$

imsart-aap ver. 2011/05/20 file: Lepinette-Tran.tex date: April 13, 2012 
Indeed, we check that Condition $(A)$ page $108[6]$ is satisfied by the sde $(\hat{\mathbf{S}})$. First, we have $\left|\widehat{\sigma}^{*}(t, x) x\right| \leq C|x|$ and secondly:

$$
\left|\widehat{\sigma}^{*}(t, x) x-\widehat{\sigma}^{*}(t, \bar{x}) \bar{x}\right| \leq\left|\widehat{\sigma}^{*}(t, x)\right||x-\bar{x}|+\left|\bar{x}\left(\widehat{\sigma}^{*}(t, x)-\widehat{\sigma}^{*}(t, \bar{x})\right)\right|
$$

where a Taylor expansion yields

$$
\widehat{\sigma}^{*}(t, x)-\widehat{\sigma}^{*}(t, \bar{x})=\widehat{\sigma}_{x}^{*}\left(t, x_{0}\right)(x-\bar{x}), \quad x_{0} \in[x, \bar{x}] .
$$

Then,

$$
\left|\bar{x}\left(\widehat{\sigma}^{*}(t, x)-\widehat{\sigma}^{*}(t, \bar{x})\right)\right| \leq\left|\widehat{\sigma}^{*}(t, x)-\widehat{\sigma}^{*}(t, \bar{x})\right|\left|\bar{x}-x_{0}\right|+\left|x_{0} \widehat{\sigma}_{x}^{*}\left(t, x_{0}\right)\right||x-\bar{x}|
$$

where $\left|x_{0} \widehat{\sigma}_{x}^{*}\left(t, x_{0}\right)\right|$ is bounded by virtue of Assumption (E). It follows that there exists a constant such that for all $x$

$$
\left|\widehat{\sigma}^{*}(t, x) x-\widehat{\sigma}^{*}(t, \bar{x}) \bar{x}\right| \leq C|x-\bar{x}| .
$$

As $\sigma_{x}$ is continuous, we conclude that Condition $(A)$ holds.

Furthermore, we have:

$$
\frac{\partial \widehat{S}_{x, t}(u)}{\partial x}=1+\int_{t}^{u} \frac{\widehat{\Lambda}^{*}\left(s, \widehat{S}_{x, t}(s)\right)}{\widehat{\sigma}^{*}\left(s, \widehat{S}_{x, t}(s)\right)} \frac{\partial \widehat{S}_{x, t}(s)}{\partial x} d W_{s}
$$

which is a strictly positive martingale (see Lemma 5.4). In the proof Lemma 4.2 , it is shown that the distribution of $\widehat{S}_{x_{0}, t}\left(\gamma_{n}\right)$ is of density with respect to the Lebesgue measure. We deduce that, out of the null-set $\widehat{S}_{x_{0}, t}\left(\gamma_{n}\right) \in$ $\left\{K_{p}: p \in N^{*}\right\}$, we have almost surely:

$$
\int_{0}^{\gamma_{n}} h^{\prime}\left(\widehat{S}_{x_{0}, t}\left(\gamma_{n}\right)+\mu\left(\widehat{S}_{x_{n}, t}\left(\gamma_{n}\right)-\widehat{S}_{x_{0}, t}\left(\gamma_{n}\right)\right) d \mu \rightarrow h^{\prime}\left(\widehat{S}_{x_{0}, t}\left(\gamma_{n}\right)\right)\right.
$$

provided that $x_{n}$ is sufficiently near to $x_{0}$ and $x_{n}$ is a subsequence such that (4.4) holds. Since $h^{\prime}$ is bounded, it follows that

$$
\widehat{u}_{x}(t, x)=E h^{\prime}\left(\widehat{S}_{x, t}\left(\gamma_{n}\right)\right) \frac{\partial \widehat{S}_{x, t}\left(\gamma_{n}\right)}{\partial x} .
$$

Finally, we define $d \bar{P}=\frac{\partial \widehat{S}_{x, t}\left(\gamma_{n}\right)}{\partial x} d P$ so that $\widehat{u}_{x}(t, x)=\bar{E} h^{\prime}\left(\widehat{S}_{x, t}\left(\gamma_{n}\right)\right)$. By virtue of the Girsanov theorem ((5.1) p 190 [8]), the process

$$
B_{u}=W_{u}-W_{t}-\int_{t}^{u} \frac{\widehat{\Lambda}^{*}\left(s, \widehat{S}_{x, t}(s)\right)}{\widehat{\sigma}^{*}\left(s, \widehat{S}_{x, t}(s)\right)} d u
$$

imsart-aap ver. 2011/05/20 file: Lepinette-Tran.tex date: April 13, 2012 
is a standard brownian motion under $\bar{P}$. Moreover, $\widehat{S}_{x, t}$ verifies the eds

(4.5) $d \widehat{S}_{x, t}(u)=\widehat{\sigma}^{*}\left(u, \widehat{S}_{x, t}(u)\right) \widehat{S}_{x, t}(u) d B_{u}+\widehat{\Lambda}^{*}\left(t, \widehat{S}_{x, t}(u)\right) \widehat{S}_{x, t}(u) d u$

Since $\widehat{\sigma}^{*}$ and $\widehat{\Lambda}^{*}$ are bounded, (4.5) admits a unique strong solution, hence a unique weak solution. We conclude that $\widehat{u}_{x}(t, x)=E h^{\prime}\left(\widetilde{S}_{x, t}\left(\gamma_{n}\right)\right)$.

Let us now give an explicit representation of $\widehat{u}_{x}(t, x)$ using the notion of fundamental solution of a PDE of parabolic type.

Lemma 4.2. We have:

$$
\widehat{u}_{x}(t, x)=\int_{-\infty}^{\infty} h^{\prime}\left(e^{z}\right) \Gamma^{*}\left(x, t, z, \gamma_{n}\right) d z
$$

where $\Gamma^{*}(x, t, z, \tau)$ is the fundamental solution of the operator:

$$
\frac{1}{2} \widehat{\sigma}_{a}^{2}(t, x) \frac{\partial^{2}}{\partial x^{2}}+\widehat{\sigma}_{b}(t, x) \frac{\partial}{\partial x}+\frac{\partial}{\partial t}
$$

and

$$
\begin{aligned}
& \widehat{\sigma}_{a}(t, x)=\widehat{\sigma}^{*}\left(t, e^{x}\right) \\
& \widehat{\sigma}_{b}(t, x)=\widehat{\Lambda}^{*}\left(t, e^{x}\right)-\frac{1}{2}\left(\widehat{\sigma}^{*}\right)^{2}\left(t, e^{x}\right) .
\end{aligned}
$$

Proof. Let us define the process $\widehat{\eta}_{x, t}(u)=\ln \widetilde{S}_{e^{x}, t}(u)$. It satisfies the following SDE:

$$
\left(\mathbf{f}^{\prime}\right)=\left\{\begin{aligned}
d \widehat{\eta}_{x, t}(u) & =\widehat{\sigma}_{a}\left(u, \widehat{\eta}_{x, t}(u)\right) d W_{u}+\widehat{\sigma}_{b}\left(u, \widehat{\eta}_{x, t}(u)\right) d u \\
\widehat{\eta}_{x, t}(t) & =x
\end{aligned}\right.
$$

To see it, it suffices to apply the Ito formula with the process $\exp \left(\widehat{\eta}_{x, t}\right)$ where $\widehat{\eta}_{x, t}$ is the solution of (f'). By virtue of Lemma 5.5, $\widehat{\eta}_{x, t}$ is a Markov process of transition density function $\Gamma^{*}\left(x, t, z, \gamma_{n}\right)$, the fundamental solution of the operator:

$$
\frac{1}{2} \widehat{\sigma}_{a}^{2}(t, x) \frac{\partial^{2}}{\partial x^{2}}+\widehat{\sigma}_{b}(t, x) \frac{\partial}{\partial x}+\frac{\partial}{\partial t} .
$$

This means that: $P\left(\widehat{\eta}_{x, t}(u) \in d z\right)=\Gamma^{*}(x, t, z, u) d z$ hence

$$
\widehat{C}_{x}(t, x)=\int_{-\infty}^{\infty} h^{\prime}\left(e^{z}\right) \Gamma^{*}\left(\ln x, t, z, \gamma_{n}\right) d z
$$


Let us now consider the special case $\alpha=0$. With Assumption $\left(\mathbf{H}^{\prime}\right)$, the function $h$ is assumed to be of the form $h(x)=h_{1}(x)+a x+b$. As $\widehat{S}_{s, x}(u)$ is a martingale, Lemma 4.1 yields

$$
\widehat{u}(s, x)=E h_{1}\left(\widehat{S}_{s, x}\left(\gamma_{n}\right)\right)+a x+b .
$$

Using the same arguments as in Lemma 4.2 , the function $E h_{1}\left(\widehat{S}_{s, x}\left(\gamma_{n}\right)\right)$ has also an explicit representation, and so is $\widehat{u}(s, x)$, which is given by the following lemma.

Lemma 4.3. Assume that Assumption (H') holds. Then,

$$
\widehat{u}(t, x)=\int_{-\infty}^{\infty} h_{1}\left(e^{z}\right) \Gamma^{*}\left(x, t, z, \gamma_{n}\right) d z+a x+b
$$

where $\Gamma^{*}(x, t, z, \tau)$ is the fundamental solution of the operator:

$$
\frac{1}{2} \widehat{\sigma}_{a}^{2}(t, x) \frac{\partial^{2}}{\partial x^{2}}+\widehat{\sigma}_{b}(t, x) \frac{\partial}{\partial x}+\frac{\partial}{\partial t}
$$

and

$$
\widehat{\sigma}_{a}(t, x)=\widehat{\sigma}\left(t, e^{x}\right), \quad \widehat{\sigma}_{b}(t, x)=-\frac{1}{2} \widehat{\sigma}^{2}\left(t, e^{x}\right)
$$

We now aim to estimate the fundamental solution $\Gamma^{*}\left(x, t, z, \gamma_{n}\right)$. To do so we use the bounds given by Theorem 8, p.263 [7]. Notice that we only need the differentiability of $\sigma(t, x)$ up to order $n=3$. In particular, we recall that

$$
\left|\frac{\partial^{k+r}}{\partial x^{k} \partial t^{r}} \Gamma^{*}\left(x, t, \xi, \gamma_{n}\right)\right| \leq \frac{C^{\prime}}{\rho_{n}(t)^{\frac{k+2 r}{2}}} \exp \left\{-C \frac{|x-\xi|^{2}}{\rho_{n}(t)}\right\}
$$

where $C, C^{\prime}$ are two positive constants which, because of the change of time, do not depend on $n$ and $\rho_{n}(t)=\gamma_{n}-t$.

We then obtain explicit expressions of the derivatives of $\widehat{C}(t, x)$. According to Lemma 4.3, we have

$$
\widehat{u}_{x}(t, x)=\int_{-\infty}^{\infty} h^{\prime}\left(e^{y}\right) \Gamma^{*}\left(\ln x, t, y, \gamma_{n}\right) d y
$$


and by the change of variable $z=e^{y}$, we obtain:

$$
\begin{aligned}
\widehat{u}_{x}(t, x) & =\int_{0}^{\infty} \frac{h^{\prime}(z)}{z} \Gamma^{*}\left(\ln x, t, \ln y, \gamma_{n}\right) d z \\
\widehat{u}_{x x}(t, x) & =\frac{1}{x} \int_{0}^{\infty} \frac{h^{\prime}(z)}{z} \frac{\partial}{\partial x} \Gamma^{*}\left(\ln x, t, \ln y, \gamma_{n}\right) d z \\
\widehat{u}_{x x x}(t, x) & =-\frac{1}{x} \widehat{u}_{x x}(t, x)+\frac{1}{x^{2}} \int_{0}^{\infty} \frac{h^{\prime}(z)}{z} \frac{\partial^{2}}{\partial x^{2}} \Gamma^{*}\left(\ln x, t, \ln y, \gamma_{n}\right) d z, \\
\widehat{u}_{x x x x}(t, x) & =-\frac{1}{x^{2}} \widehat{u}_{x x}(t, x)-\frac{3}{x} \widehat{u}_{x x x}(t, x)+\frac{1}{x^{2}} \int_{0}^{\infty} \frac{h^{\prime}(z)}{z} \frac{\partial^{3}}{\partial x^{3}} \Gamma^{*}\left(\ln x, t, \ln y, \gamma_{n}\right) d z, \\
\widehat{u}_{x t}(t, x) & =\int_{0}^{\infty} \frac{h^{\prime}(z)}{z} \frac{\partial}{\partial t} \Gamma^{*}\left(\ln x, t, \ln y, \gamma_{n}\right) d z, \\
\widehat{u}_{x x t}(t, x) & =\int_{0}^{\infty} \frac{h^{\prime}(z)}{z} \frac{\partial^{2}}{\partial t \partial x} \Gamma^{*}\left(\ln x, t, \ln y, \gamma_{n}\right) d z .
\end{aligned}
$$

Using the bounds given by (4.6) and the relations (4.1), we immediately deduce some bounds for the derivatives of $\widehat{C}(t, x)$ which are given in the following theorem.

Theorem 4.4. There exists some constants $A, C>0$ independent of $n$ such that:

$$
\begin{aligned}
\left|\widehat{C}_{x x}(t, x)\right| & \leq \frac{C}{x \rho_{t}}, \\
\left|\widehat{C}_{x x x}(t, x)\right| & \leq \frac{C}{x^{2} \rho_{t}}+\frac{C}{x^{2} \rho_{t}^{2}}, \\
\left|\widehat{C}_{x x x x}(t, x)\right| & \leq \frac{C}{x^{2} \rho_{t}}+\frac{C}{x^{2} \rho_{t}^{2}}+\frac{C}{x^{2} \rho_{t}^{3}}, \\
\left|\widehat{C}_{x t}(t, x)\right| & \leq \frac{C}{1-t}, \\
\left|\widehat{C}_{x x t}(t, x)\right| & \leq \frac{C \gamma_{n}}{x \rho_{t}^{3}},
\end{aligned}
$$

where $\rho_{t}^{2}:=\gamma_{n}(1-t)$.

We conclude this section with a specific bound we need in the case $\alpha=0$ under Assumption (H'). As $u(s, x)$ has a probabilistic representation given by Lemma 4.3) where the function $h_{1}$ is bounded, we get a bound for $\widehat{C}_{x t}(t, x)$ which is comparable to that of $\widehat{C}_{x x t}(t, x)$ in the case $\alpha>0$. Indeed, recall 
that the bound we obtain for $\widehat{C}_{x x t}(t, x)$ is deduced from the probabilistic representation of $u_{x}(s, x)$. Therefore, we have

Lemma 4.5. Suppose that Assumption (H') holds. Then,

$$
\left|\widehat{C}_{x t}(t, x)\right| \leq \frac{C \gamma_{n}}{x \rho_{t}^{3}}
$$

where $\rho_{t}^{2}:=\gamma_{n}(1-t)$.

\section{Appendix.}

Lemma 5.1. The stochastic equation defined on $[s,+\infty[$ for all $s>0$ by:

$$
(\hat{\mathbf{S}})=\left\{\begin{aligned}
d \widehat{S}_{x, s}(t) & =\widehat{\sigma}\left(t, \widehat{S}_{x, s}(t)\right) \widehat{S}_{x, s}(t) d W_{t} \\
\widehat{S}_{x, s}(s) & =x
\end{aligned}\right.
$$

has a unique solution such that for some constant $C^{*}=C^{*}(n, T)$,

$$
E \sup _{0 \leq t \leq T} \widehat{S}_{x, s}^{2}(t) \leq C^{*}\left(1+x^{2}\right)
$$

Proof. It suffices to apply Theorem 2.2 p104 [6].

Lemma 5.2. Assume that Condition (H) holds. Then, the PDE (e) has a unique solution.

Proof. Note that it is not possible to conclude immediately on the existence of a solution of (e) because the operator is not uniformly parabolic on ] $0, \infty[\otimes[0,1[$. This is why, we transform the problem in such a way that the required uniform parabolic condition holds.

By virtue of Lemma 5.1, recall that $\widehat{S}_{x, s}(t)$ is the unique solution of the stochastic equation defined on $[s, 1], s \in[0,1]$ by :

$$
\left\{\begin{aligned}
d \widehat{S}_{x, s}(t) & =\widehat{\gamma}\left(t, \widehat{S}_{x, s}(t)\right) d W_{t} \\
\widehat{S}_{x, s}(s) & =x
\end{aligned}\right.
$$

where $\widehat{\gamma}(t, x)=\sqrt{\sigma^{2}(t, x) x^{2}+\sigma(t, x) x^{2} \gamma_{n}}$. Recall that

$$
E \sup _{s \leq t \leq 1} \widehat{S}_{x, s}^{2}(t) \leq C_{n}^{*}\left(1+x^{2}\right)
$$

imsart-aap ver. 2011/05/20 file: Lepinette-Tran.tex date: April 13, 2012 
where $C_{n}^{*}$ is a constant depending on $n$. Let us define $g(x, t):=E h\left(\widehat{S}_{x, t}(1)\right)$. It satisfies the inequality

$$
\begin{aligned}
|g(x, t)| & \leq c\left(1+E\left|\widehat{S}_{x, t}(1)\right|\right) \leq c\left(1+\left(E \widehat{S}_{x, t}^{2}(1)\right)^{1 / 2}\right) \\
& \leq c(1+|x|) .
\end{aligned}
$$

Since $h^{\prime}$ is bounded, we obtain by virtue of the Cauchy-Schwarz inequality, that

$$
|g(x, t)-g(y, u)| \leq c \sqrt{E\left(\widehat{S}_{x, t}(1)-\widehat{S}_{y, u}(1)\right)^{2}}
$$

Let $\widehat{S}_{x, s}^{(m)}$ be the solution of the sde

$$
\left\{\begin{aligned}
d \widehat{S}_{x, s}^{(m)}(t) & =\widehat{\gamma}^{(m)}\left(t, \widehat{S}_{x, s}^{(m)}(t)\right) d W_{t} \\
\widehat{S}_{x, s}^{(m)}(s) & =x
\end{aligned}\right.
$$

where $\widehat{\gamma}^{(m)}(t, x):=\sqrt{\sigma^{2}(t, x) x^{2}+\sigma(t, x) \gamma_{n} x^{2}+m^{-1}}$. Observe than $\| \widehat{\gamma}^{(m)}-$ $\widehat{\gamma} \|_{\infty} \leq m^{-1 / 2}$ hence $\widehat{S}_{x, s}(1)^{(m)} \rightarrow \widehat{S}_{x, s}(1)$ in $L^{2}(\Omega, P)$ as $m \rightarrow \infty$ uniformly in $x$ and $s$. Then, $g^{(m)}(x, t):=E h\left(\widehat{S}_{x, t}^{(m)}(1)\right)$ converges uniformly to $g(x, t)$. Applying Lemma 3.3 p 112 with Condition $\left(A^{\prime}\right)$ p 113 [6], we deduce the existence of a constant $C_{R}^{(m)}$ such that

$$
\left|g^{(m)}(x, t)-g^{(m)}(y, u)\right| \leq C_{R}^{(m)} \sqrt{(x-y)^{2}+|t-u|}
$$

if $|x|,|y| \leq R$. We deduce that $g^{(m)}$ is continuous hence so is $g$.

We use the notations of page 138 [6] where we replace $t$ by $1-t$. Let us consider the following sets with $m \in \mathbb{N} \backslash\{0\}$ :

$$
\begin{aligned}
Q_{m} & =] \frac{1}{m}, m[\times] 0,1[, \\
B_{m} & =] \frac{1}{m}, m[\times\{1\}, \\
T_{m} & =] \frac{1}{m}, m[\times\{0\}, \\
S_{m} & =\left\{\frac{1}{m}, m\right\} \times[0,1[.
\end{aligned}
$$

For each $y \in \partial Q_{m}$, it is easy to observe that there exists a closed ball $K_{y}^{m}$ such that $K_{y}^{m} \cap Q_{m}=\emptyset$ and $K_{y}^{m} \cap \overline{Q_{m}}=\{y\}$. It follows that the function $W_{y}$ proposed p 134 [6] defines a barrier for each $y \in S_{m} \subseteq \partial Q_{m}$. Moreover, observe that $g(x, t)=E h\left(\widehat{S}_{x, 1}(1)\right)=h(x)$ if $(x, t) \in \overline{B_{m}} \cap \overline{S_{m}}$. By virtue of 
Theorem 3.6 p 138 [6], Assumptions $(\mathbf{G})$ and $(\mathbf{P})$, implie that the Dirichlet problem

$$
\left(\mathbf{D}_{\mathbf{m}}\right)=\left\{\begin{array}{lc}
u_{t}(t, x)+\frac{1}{2} \widehat{\sigma}^{2}(t, x) x^{2} u_{x x}(t, x)=0 & (x, t) \in Q_{m} \cup T_{m} \\
u(1, x)=h(x) & x \in B_{m} \\
u(t, x)=g(x, t) & (x, t) \in S_{m}
\end{array}\right.
$$

admits a unique solution $u^{m}$. Indeed, $g$ and $h$ are continuous. Since $\overline{Q_{m}}$ is bounded, there is a constant $c_{m}$ depending on $m$ such that

$$
\left|\widehat{\gamma}^{2}(t, x)-\widehat{\gamma}^{2}(t, \bar{x})\right| \leq c_{m}|x-\bar{x}|
$$

Note that $u^{m}$ is continuous on $\overline{Q_{m}}$ and the derivatives $u_{t}^{m}, u_{x x}^{m}$ are continuous on $Q_{m} \cup T_{m}$ (see Theorem $3.6 \mathrm{p} 138$ [6]). By virtue of Theorem $5.2 \mathrm{p} 147$ [6], we deduce that that $u_{m}$ has the following stochastic representation

$$
u^{m}(x, t)=E g\left(\widehat{S}_{x, t}(\tau), \tau\right) I_{\tau<1}+\operatorname{Eh}\left(\widehat{S}_{x, t}(1)\right) I_{\tau=1},
$$

where $\tau$ is a stopping time. It follows that $u^{m}(x, t)=E g\left(\widehat{S}_{x, t}(\tau), \tau\right)$.

On the other hand,

$$
g\left(\widehat{S}_{x, t}(\tau), \tau\right)=\operatorname{Eh}\left(\widehat{S}_{\widehat{S}_{x, t}(\tau), \tau}(1)\right)
$$

where $\widehat{S}_{\widehat{S}_{x, t}(\tau), \tau}(1)=\widehat{S}_{x, t}(1)$. It follows that $u^{m}(x, t)=g(x, t)$ and, as $m \rightarrow$ $\infty$, we deduce that $g$ is a solution to the PDE (e). Indeed, it is easy to check that $g$ verifies $(\mathbf{e})$. Moreover, $v(t, y)=u\left(t, e^{y}\right)$ is a solution of the following uniformly parabolic PDE

$(\mathbf{f})=\left\{\begin{array}{l}v_{t}(t, y)+\frac{1}{2} \widehat{\sigma}^{2}\left(e^{y}\right) v_{y y}(t, y) \quad-\frac{1}{2} \widehat{\sigma}^{2}\left(e^{y}\right) v_{y}(t, y)=0, \quad(y, t) \in \mathbf{R} \times[0,1[ \\ v(1, y)=h\left(e^{y}\right), \quad x \in \mathbf{R} .\end{array}\right.$

By virtue of Theorem 3.6 [6], $v$ is also the unique solution of the same PDE restricted to an arbitrary smooth bounded domain. Moreover, by virtue of Theorem $5.2 \mathrm{p} 147$ [6], we deduce that the solution $v$ has a probabilistic representation which is unique. We then conclude that $u$ is also unique.

Lemma 5.3. Assume that $t \in\left[0, \gamma_{n}\right]$. Then, the stochastic equation:

$$
\left\{\begin{aligned}
d \widetilde{S}_{x, t}(u) & =\widehat{\sigma}^{*}\left(u, \widetilde{S}_{x, t}(u)\right) \widetilde{S}_{x, t}(u) d W_{u}+\widehat{\Lambda}^{*}\left(u, \widetilde{S}_{x, t}(u)\right) \widetilde{S}_{x, t}(u) d u \\
\widetilde{S}_{x, t}(t) & =x
\end{aligned}\right.
$$

has a unique solution on $\left[t, \gamma_{n}\right]$. 
Proof. It suffices to use Theorem 2.2 p104 [6]. For this, we verify the needed conditions with:

$$
\begin{aligned}
\widetilde{\sigma}(t, x) & =\widehat{\sigma}^{*}(t, x) x, \\
b(t, x) & =\widehat{\Lambda}^{*}(t, x) x .
\end{aligned}
$$

From

$$
\left(\widehat{\sigma}^{*}\right)^{2}(t, x)=\frac{1}{\gamma_{n}} \sigma^{2}(t, x)+\sigma(t, x)
$$

we deduce that

$$
2 \widehat{\sigma}_{x}^{*}(t, x) \widehat{\sigma}^{*}(t, x)=\frac{2}{\gamma_{n}} \sigma_{x}(t, x) \sigma(t, x)+\sigma_{x}(t, x) .
$$

Recall that

$$
\widehat{\Lambda}^{*}(t, x)=\left(\widehat{\sigma}^{*}\right)^{2}(t, x)+\widehat{\sigma}_{x}^{*}(t, x) \widehat{\sigma}^{*}(t, x) x .
$$

Then, from the hypothesis $\left|x \sigma_{x}(t, x)\right| \leq$ const it is easy to deduce that $|b(t, x)| \leq$ const $|x|$. Otherwise, it is clear that $|\widetilde{\sigma}(t, x)| \leq$ const $|x|$.

Finally, we suppose that $|x|,|\bar{x}| \leq N$. We have

$$
|b(t, x)-b(t, \bar{x})| \leq\left|\widehat{\Lambda}^{*}(t, x)\right||x-\bar{x}|+|\bar{x}|\left|\widehat{\Lambda}^{*}(t, x)-\widehat{\Lambda}^{*}(t, \bar{x})\right|
$$

where $\left|\widehat{\Lambda}^{*}(t, x)\right| \leq$ const, $|\bar{x}| \leq N$ and

$\widehat{\Lambda}^{*}(t, x)-\widehat{\Lambda}^{*}(t, \bar{x})=\left(\widehat{\sigma}^{*}\right)^{2}(t, x)-\left(\widehat{\sigma}^{*}\right)^{2}(t, \bar{x})+x \widehat{\sigma}_{x}^{*}(t, x) \widehat{\sigma}^{*}(t, x)-\bar{x} \widehat{\sigma}_{x}^{*}(t, \bar{x}) \widehat{\sigma}^{*}(t, \bar{x})$.

But we have $\left|\left(\widehat{\sigma}^{*}\right)^{2}(t, x)-\left(\widehat{\sigma}^{*}\right)^{2}(t, \bar{x})\right| \leq$ const $|x-\bar{x}|$ and

$$
2 x \widehat{\sigma}_{x}^{*}(t, x) \widehat{\sigma}^{*}(t, x)=x \frac{2}{\gamma_{n}} \sigma_{x}(t, x) \sigma(t, x)+x \sigma_{x}(t, x) .
$$

Since the next expression is bounded, we first write $x=(x-\bar{x})+\bar{x}$ and finally, we have to estimate

$$
\begin{aligned}
\sigma_{x}(t, x) \sigma(t, x)-\sigma_{x}(t, \bar{x}) \sigma(t, \bar{x})= & \sigma(t, x)\left(\sigma_{x}(t, x)-\sigma_{x}(t, \bar{x})\right) \\
& +\sigma_{x}(t, \bar{x})(\sigma(t, x)-\sigma(t, \bar{x}))
\end{aligned}
$$

where

$$
\begin{aligned}
\left|\sigma_{x}(t, x)-\sigma_{x}(t, \bar{x})\right| & \leq \text { const }|x-\bar{x}| \\
|\sigma(t, x)-\sigma(t, \bar{x})| & \leq \text { const }|x-\bar{x}|
\end{aligned}
$$

imsart-aap ver. 2011/05/20 file: Lepinette-Tran.tex date: April 13, 2012 
because $\sigma_{x x}(t, x)$ is bounded. Then, we can conclude that for $|x|,|\bar{x}| \leq N$,

$$
|b(t, x)-b(t, \bar{x})| \leq \operatorname{const}(N)|x-\bar{x}|
$$

In a similar way, it is easy to prove that

$$
|\widetilde{\sigma}(t, x)-\widetilde{\sigma}(t, \bar{x})| \leq \operatorname{const}(N)|x-\bar{x}|
$$

\section{Lemma 5.4. The local martingale}

$$
\frac{\partial \widehat{S}_{x, t}(u)}{\partial x}=1+\int_{t}^{u} \frac{\widehat{\Lambda}^{*}\left(s, \widehat{S}_{x, t}(s)\right)}{\widehat{\sigma}\left(s, \widehat{S}_{x, t}(s)\right)} \frac{\partial \widehat{S}_{x, t}(s)}{\partial x} d W_{s}
$$

is a strictly positive martingale on any interval $[t, T] \subseteq[0, \infty)$.

Proof. The Doleans-Dade formula give us

$$
\frac{\partial \widehat{S}_{x, t}(u)}{\partial x}=\exp \left\{\int_{t}^{1} \widehat{\Lambda}^{*}\left(v, \widehat{S}_{x, t}(v)\right) d W_{v}-\frac{1}{2} \int_{t}^{1}\left(\widehat{\Lambda}^{*}\right)^{2}\left(v, \widehat{S}_{x, t}(v)\right) d v\right\} .
$$

Since $\widehat{\Lambda}^{*}$ is bounded, we deduce that there exists a constant $c$ such that

$$
\left(\frac{\partial \widehat{S}_{x, t}(u)}{\partial x}\right)^{2} \leq c N_{u}
$$

where

$$
N_{u}=\exp \left\{\int_{t}^{u} 2 \widehat{\Lambda}^{*}\left(v, \widehat{S}_{x, t}(v)\right) d W_{v}-\frac{1}{2} \int_{t}^{1} 4\left(\widehat{\Lambda}^{*}\right)^{2}\left(v, \widehat{S}_{x, t}(v)\right) d v\right\}
$$

is a strictly positive locale martingale, hence a supermartingale verifying

$$
d N_{u}=2 N_{u} \widehat{\Lambda}^{*}\left(u, \widehat{S}_{x, t}(u)\right) d W_{u}
$$

In particular $N$ is integrable and finally

$$
\sup _{u \in[t, T]} E\left(\frac{\partial \widehat{S}_{x, t}(u)}{\partial x}\right)^{2}<\infty .
$$

So, we can conclude about the lemma.

imsart-aap ver. 2011/05/20 file: Lepinette-Tran.tex date: April 13, 2012 
Lemma 5.5. The process $\widehat{\eta}_{x, t}$ is a Markov process of transition density function $\Gamma^{*}(x, t, z, \tau)$, the fundamental solution of the operator:

$$
\frac{1}{2} \widehat{\sigma}_{a}^{2}(t, x) \frac{\partial^{2}}{\partial x^{2}}+\widehat{\sigma}_{b}(t, x) \frac{\partial}{\partial x}+\frac{\partial}{\partial t} .
$$

Proof. According to Theorem 5.4 p 149 [6], it suffices to verify the needed conditions. Condition $\left(A_{1}\right)$ is well verified since $\widehat{\sigma}_{a}^{2}(t, x) \geq$ const $>0$. Let us verify Condition $\left(B_{1}\right)(i)$ : First, $\widehat{\sigma}_{a}^{2}(t, x)=\left(\widehat{\sigma}^{*}\right)^{2}(t, x)$ and $\widehat{\sigma}_{b}(t, x)$ are bounded. Secondly, suppose that $|x|,|\bar{x}| \leq N$. Then

$$
\left|\widehat{\sigma}^{2}\left(t, e^{x}\right)-\widehat{\sigma}^{2}\left(t^{\prime}, e^{\bar{x}}\right)\right| \leq\left|\left(\widehat{\sigma}^{*}\right)^{2}\left(t, e^{x}\right)-\left(\widehat{\sigma}^{*}\right)^{2}\left(t^{\prime}, e^{x}\right)\right|+\left|\left(\widehat{\sigma}^{*}\right)^{2}\left(t^{\prime}, e^{x}\right)-\left(\widehat{\sigma}^{*}\right)^{2}\left(t^{\prime}, e^{\bar{x}}\right)\right|
$$

where, as already shown, $\left|\left(\widehat{\sigma}^{*}\right)^{2}\left(t^{\prime}, e^{x}\right)-\left(\widehat{\sigma}^{*}\right)^{2}\left(t^{\prime}, e^{\bar{x}}\right)\right| \leq c|x-\bar{x}|$. Moreover,

$\left|\left(\widehat{\sigma}^{*}\right)^{2}\left(t, e^{x}\right)-\left(\widehat{\sigma}^{*}\right)^{2}\left(t^{\prime}, e^{x}\right)\right| \leq \frac{1}{\gamma_{n}}\left|\sigma^{2}\left(t, e^{x}\right)-\sigma^{2}\left(t^{\prime}, e^{x}\right)\right|+\left|\sigma\left(t, e^{x}\right)-\sigma\left(t^{\prime}, e^{x}\right)\right|$.

It follows that if $|x|,|\bar{x}| \leq N$,

$$
\left|\widehat{\sigma}_{a}^{2}\left(t, e^{x}\right)-\widehat{\sigma}_{a}^{2}\left(t^{\prime}, e^{\bar{x}}\right)\right| \leq C(N)\left(\left|t-t^{\prime}\right|+|x-\bar{x}|\right) .
$$

In a similar way, since we suppose that $\widehat{\sigma}_{x, t}$ is bounded, we have

$$
\left|\widehat{\sigma}_{b}\left(t, e^{x}\right)-\widehat{\sigma}_{b}\left(t^{\prime}, e^{\bar{x}}\right)\right| \leq \operatorname{const}(N)\left(\left|t-t^{\prime}\right|+|x-\bar{x}|\right) .
$$

Finally, since $x \widehat{\sigma}_{x}(t, x)$ is bounded, we deduce that Condition $\left(B_{1}\right)(i i)$ holds, i.e. for any $x, \bar{x}$,

$$
\left|\widehat{\sigma}_{a}^{2}\left(t, e^{x}\right)-\widehat{\sigma}_{a}^{2}\left(t, e^{\bar{x}}\right)\right| \leq C(|x-\bar{x}|)
$$

Lemma 5.6. Assume that $h$ is a convex function verifying Condition $\mathbf{H}$. If $\sigma$ does not depend on $t$, then $\widehat{C}_{x x} \geq 0$.

According to the Tanaka-Meyer formula, we have:

$$
h\left(\widehat{S}_{x, t}(1)\right)=h(x)+\int_{t}^{1} h_{-}^{\prime}\left(\widehat{S}_{x, t}(u)\right) d W_{u}+\frac{1}{2} \int_{\mathbb{R}} L_{1}^{u} \mu(d u)
$$

where $h_{-}^{\prime}$ is the left derivative and

$$
\mu=h^{\prime \prime}(u) d u+\sum_{i}\left[h_{+}^{\prime}\left(K_{i}\right)-h_{+}^{\prime}\left(K_{i}\right)\right] \delta_{K_{i}}
$$

imsart-aap ver. 2011/05/20 file: Lepinette-Tran.tex date: April 13, 2012 
$\delta_{K_{i}}$ is the Dirac measure. Moreover, $\left(L_{s}^{u}\right)_{s \in[t, 1]}$ is a continuous and positive semi-martingale verifying

$$
\int_{\mathbb{R}} g(u) L_{s}^{u} d u=\int_{t}^{s} g\left(\widehat{S}_{x, t}(u)\right) d\left\langle\widehat{S}_{x, t}\right\rangle_{u}, \quad s \in[t, 1]
$$

for any positive and bounded measurable function $g$. It follows that

$$
\begin{aligned}
h\left(\widehat{S}_{x, t}(1)\right) & =h(x)+\int_{t}^{1} h_{-}^{\prime}\left(\widehat{S}_{x, t}(u)\right) d W_{u}+\frac{1}{2} \sum_{i}\left[h_{+}^{\prime}\left(K_{i}\right)-h_{-}^{\prime}\left(K_{i}\right)\right] L_{1}^{K_{i}} \\
(5.1)+ & +\frac{1}{2} \int_{t}^{1} h^{\prime \prime}\left(\widehat{S}_{x, t}(u)\right) \widehat{\sigma}^{2}\left(\widehat{S}_{x, t}(u)\right) \widehat{S}_{x, t}^{2}(u) d u .
\end{aligned}
$$

Recall that

$$
\left(\widehat{S}_{x, t}(u)-K\right)^{+}=(x-K)^{+}+\int_{t}^{u} I_{\widehat{S}_{x, t}(s)>K} d \widehat{S}_{x, t}(s)+\frac{1}{2} L_{u}^{K} .
$$

Then,

$$
\frac{1}{2} E L_{1}^{K_{i}}=\widehat{C}_{i}(t, x)-\left(x-K_{i}\right)^{+}
$$

where $\widehat{C}^{i}(t, x)$ is the solution of (e) when $h(x)=\left(x-K_{i}\right)^{+}$. Taking the expectations, we deduce from (5.1) that

$$
\widehat{C}_{t}(t, x)=\sum_{i} \alpha_{i} \widehat{C}_{t}^{i}(t, x)-\frac{1}{2} E\left(h^{\prime \prime}\left(\widehat{S}_{x, t}(1)\right) \widehat{\sigma}^{2}\left(\widehat{S}_{x, t}(1)\right) \widehat{S}_{x, t}^{2}(1)\right)
$$

where $\alpha_{i}=h_{+}^{\prime}\left(K_{i}\right)-h_{+}^{\prime}\left(K_{i}\right) \geq 0$. Indeed, to differentiate (5.1) with respect to $t$, we note that $\widehat{S}_{x, t}(u)=\bar{S}_{x, 0}(u-t)$ where $\bar{S}_{x, 0}$ verifies

$$
d \bar{S}_{x, 0}(v)=\widehat{\sigma}\left(\bar{S}_{x, 0}(v)\right) \bar{S}_{x, 0}(v) d W_{v}, \quad v \in[0,1-t]
$$

and we use the change of variable $v=u-t$. Since $h^{\prime \prime} \geq 0$ and

$$
\widehat{C}_{t}(t, x)=-\frac{1}{2} \widehat{\sigma}^{2}(t, x) x^{2} \widehat{C}_{x x}(t, x)
$$

it is then sufficient to show the lemma for $h(x)=(x-K)^{+}$. To do so, we define:

$$
\begin{aligned}
h_{n}(x) & :=0 \quad x \in[0, K-1 / n] \\
& :=n(x-K+1 / n)^{2} / 4 \quad x \in[K-1 / n, K+1 / n] \\
& :=x \quad x \in[K+1 / n, \infty[.
\end{aligned}
$$


The function $h_{n}$ is a continuous and convex function which satisfies the inequalities

$$
\begin{aligned}
& 0 \leq h_{n}(x)-h(x) \leq \frac{1}{4 n}, \\
& \left|h_{n}^{\prime}(x)-h^{\prime}(x)\right| \leq I_{[K-1 / n, K+1 / n]}(x)
\end{aligned}
$$

It follows that $\widehat{C}_{x}^{n}(t, x) \rightarrow \widehat{C}_{x}(t, x)$ where $\widehat{C}^{n}, \widehat{C}$ are the solutions of (e) respectively when the terminal conditions are given by $h_{n}$ and $h(x)=(x-$ $K)^{+}$. Since $h_{n}$ is a $C^{1}$-function, the coefficients $\alpha_{i}=0$ and we deduce from (5.2) that $\widehat{C}_{x x}^{n}(t, x) \geq 0$ and $x \rightarrow \widehat{C}_{x}^{n}$ is increasing. Then, $x \rightarrow \widehat{C}_{x}$ is also increasing and finally $\widehat{C}_{x x} \geq 0$.

\section{References.}

[1] Bouchouev I. and Isakov V. Uniqueness, stability and numerical methods for the inverse problem that arises in financial markets. Inverse Problems Vol 15, 3 (1999).

[2] Denis E. Marchés avec Coûts de Transactions: Approximation de Leland et Arbitrage. Thesis, University of Franche-Comté (2008). ${ }^{1}$

[3] Lépinette E., Modified Leland's Strategy for Constant Transaction Costs Rate. Mathematical Finance (2012).

[4] Denis-Lépinette E. Approximate Hedging of Contingent Claims under Transaction Costs. Applied Mathematical Finance, 17 (2010).

[5] Egger H., Hein T. and Hofmann B. On decoupling of volatility smile and term structure in inverse option pricing. Inverse Problems Vol 22, 4 (2006).

[6] Friedman A. Stochastic Differential Equations and Applications. Volume 1. Academic Press ( 1975).

[7] Friedman, A. Partial differential equations of parabolic type. Prentice-Hall, Inc., Englewood Cliffs, N.J. 347 pp (1964).

[8] Karatzas.I, Shreve.S.E. Brownian Motion and Stochastic Calculus. Springer Verlag.

[9] Leland H. Option pricing and Replication with Transactions Costs, Journal of Finance, XL, 5, 1283-1301 (1985).

[10] Lions P.L, Musiela M. Convexity of Solutions of Parabolic Equations. ELSVIER. C.R.Acad.Sci.Paris, Ser.I 342,915-921 (2006).

[11] Claude Martini. Propagation of Convexity by Markovian and Martingalian Semigroups. Potential Analysis 10: 133-175. Kluwer Academic Publishers (1999).

Ceremade, Paris-Dauphine University, Place du Maréchal De lattre De Tassigny, 75775 Paris Cedex 16, France

E-MAIL: emmanuel.lepinette@ceremade.dauphine.fr; tran@ceremade.dauphine.fr.

\footnotetext{
${ }^{1}$ Available on http://artur.univ-fcomte.fr/ST/MATHAPP/these/denis.pdf 